\title{
Heart disease complicating pregnancy - management strategies
}

\author{
Priyadharshan $\mathrm{P}^{1}$
}

\section{Introduction}

Heart disease is the third most common cause of maternal death and the leading non-obstetric cause of maternal deaths. Cardiac patients need a stable haemodynamic condition throughout pregnancy. During pregnancy, changing haemodynamics exacerbate cardiac symptoms, in worse scenario can be even be life threatening to mother and unborn baby. In some instances healthy mothers become cardiac patients during the course of pregnancy (eg. postpartum cardiomyopathy). Antenatal history taking and cardiac examination during first visit in all pregnant women is essential as some of the preexisting heart conditions can only be screened and picked up at that point.

\section{Haemodynamic modifications during pregnancy}

Hormonal changes during pregnancy relax vascular smooth muscles and determine the increase blood volume which starts to rise as early as the fifth week. This rise reaches 50\% towards the end of pregnancy. Both systemic vascular resistance and blood pressure decrease and the resting heart rate increases $10-20$ beats per minute. The result is an increase of $30-50 \%$ in cardiac output, which is mainly achieved by an increase in stroke volume. During labour and delivery a further increase in cardiac output and blood pressure is evident particularly during uterine contractions. During early post partum period cardiac output is increased because

${ }^{1}$ Resident Consultant Cardiologist, Cardiology Unit, National Hospital of Sri Lanka, Colombo.

\section{Correspondence: P Priyadharshan}

E-mail: prakash.priyadharshan@gmail.com

Competing interests: None
Figure 1. Changes in haemodynamic parameters with gestational age (Adapted from the American College of Cardiology Foundation).

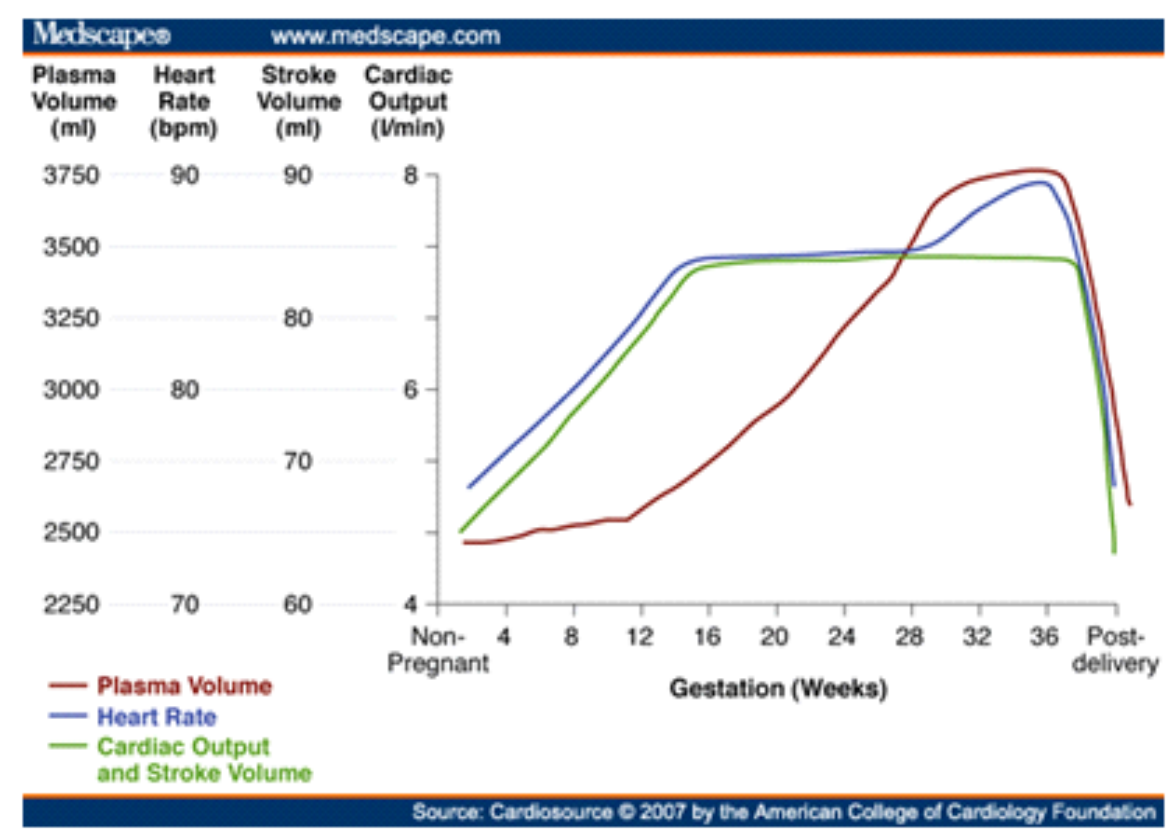

additional blood volume reaches the circulation from the contracting uterus. These changes could precipitate pulmonary oedema in high risk patients. Haemodynamic conditions have largely returned to normal within 1-3 days in most cases but may take up to a week.

\section{Basic concepts in management}

Management of pregnant mothers with cardiac disease involves understanding haemodynamics in various stages of the pregnancy.

\section{Pre conception}

All cardiac patients need to be assessed according to the New York Heart Association classification (NYHA) and should be categorized as high risk when NYHA functional class 3-4 symptoms present. Usually they don't have enough cardiac reserve to withstand the haemodynmic stress especially when it is associated with severe pulmonary hypertension, severe left ventricular outflow tract obstruction like aortic stenosis and cyanotic heart disease. Pregnancy is contraindicated in this category of patients. Termination of pregnancy could be considered after counseling as the risks to the mother are high (mortality 8-35\%, morbidity 50\%). To prevent pregnancy apart from barrier methods (condom), the levonorgestrel releasing intrauterine device or implants are safe and effective contraceptive that can be used in women with cyanotic congenital heart disease and pulmonary vascular disease. SABE prophylaxis is not routinely recommended for IUCD insertion.Preconceptional genetic counseling should be given to parents with suspected hereditary heart disease. 


\section{During pregnancy}

Patients with cardiac diseases need close follow-up usually after the beginning of the second trimester. In patient management could be considered in high risk patients. Primary aim should be to optimize haemodynamic status for delivery and this could be achieved by medication but they should be safely non-teratogenic. Antiplatelet agents aspirin is well tolerated and safe during pregnancy. Clopidogrel has limited information on safety but could be considered when benefits outweigh the risk. Rate controlling medications such as beta blocker can cause fetal bradycardia and hypoglycemia, diltiazam has possible teratogenic effect, digoxin is safe but monitoring dioxin levels is unreliable. ACE inhibitors and angiotensin receptor blockers are contraindicated as they can cause renal dysplasia. Stations, atorvastatin, rosuvastatin, also can cause congenital anomalies and are contraindicated in pregnancy. Anticoagulants such as wafarin can cause embryopathy when used in first trimester but it is dose dependent. Heparin is safe but long term usage can cause osteoporosis. Anti arrthymic medication amiadarone can cause fetal hypo, hyperthyroidism.

In case of failed medical management cardiac interventions could be performed for optimization for delivery. In order to prevent possible further aggravation of cardiac status any intervention should be planned in the 2nd trimester. Procedure related complication to mother and foetus has to be minimized. Percutaneous interventions such as percutaneous transeptal mitral commissurotomy (PTMC) for severe mitral stenosis, aortic valvuloplasty for severe aortic stenosis are performed during second trimester. Open surgeries such as valve repair and coronary artery bypass graft also can be performed during second trimester. Since open cardiac surgeries have high fetal mortality, strict fetal monitoring with umbilical artery Doppler during surgery is recommended and in order to change maternal cardiac settings in cardiac bypass (pulsatile/ non pulsatile) cardiac anesthetist should be informed when there is a compromise in fetal blood flow. Radiation on the foetus depends on the radiation dose and the gestational age at which exposure occurs. If possible, procedures should be delayed until at least the completion of the period of major organogenesis (12 weeks after menses). The safe dose is $<50 \mathrm{mGy}$.

\section{During delivery}

Mild to moderate risk patients can undergo normal vaginal delivery. Second stage should be assisted to reduce the labour time and pain should be controlled with epidural anesthesia. Hemodynamic monitoring is vital throughout labour and cardiac patients ideally should be managed in a high dependency unit or in an ICU. High risk patients may not withstand the stress of labour. These patients should undergo planned cesarean section when the foetus considered viable. Even termination of pregnancy has its attendant risks of $7 \%$ maternal mortality in high risk patients because of vasodilatation and depression of myocardial contractility due to anesthesia.

Endocarditis prophylaxis is now only recommended for patients at highest risk of aquiring endocarditis during high risk procedures, eg. dental procedures. During delivery the indication for prophylaxis has been controversial and, given the lack of convincing evidence that infective endocarditis is related to either vaginal or caesarean delivery, antibiotic prophylaxis is not recommended during vaginal or caesarean delivery.

\section{Post delivery}

Monitoring patient in post delivery is important. The plasma volume and cardiac output increase during post partum period due to uterine contraction. Mothers can develop symptoms if they have poor cardiac reserve. It can be managed with standard cardiac medications inclu- ding diuretics. In high risk patients reliable contraceptive methods should be discussed before discharge. Genetic counseling also should be given to the mother before considering future pregnancy in case of family history of congenital cardiac disease.

\section{Specific cardiac conditions}

\section{Pulmonary embolism}

It is the leading non-obstetric cause of maternal mortality. The most dangerous period still being the first 24 hours after delivery (vaginal or surgical), and the heightened risk continuing although diminishing over the next 6 weeks. The clinical symptoms and signs of pulmonary embolism during pregnancy include dyspnoea, chest pain, tachycardia, haemoptysis, and collapse. Diagnosis is made by clinical suspicion and aided by investigations. Compression ultrasonography of leg veins to exclude DVT can be considered. $\mathrm{D}$ dimers could be positive in pregnancy therefore, it cannot be used solely to diagnose pulmonary embolism during pregnancy. MRI does not involve radiation exposure and not harmful to the fetus. It has a high sensitivity and specificity for the diagnosis of iliac vein thrombosis.

\section{Figure 2. CT scan-saddle shaped thrombi in main pulmonary artery.}

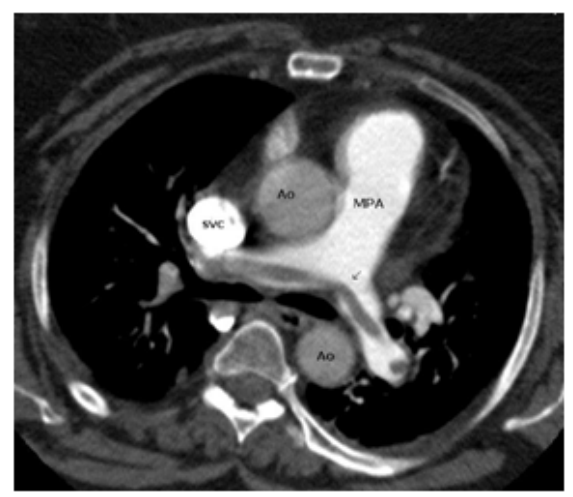

CT pulmonary angiography should be performed, when the diagnosis cannot be confirmed (Figure 2). It is preferred over ventilation-perfusion lung scanning 
for the diagnosis of pulmonary embolism because radiation dose to foetus is comparatively high in perfusion scan still lower than cut off value for dangerous level to the foetus.

High risk patients with low blood pressure need streptokinase although it is relatively contraindicated. Other patients can be managed with heparin infusion. In patients with recent pulmonary embolism, pre-partum heparin treatment should be restarted $6 \mathrm{~h}$ after a vaginal birth and $12 \mathrm{~h}$ after a caesarean delivery. To prevent pulmonary embolism all patients should be mobilized early and well hydrated. High risk patients should use compression stockings with LMW heparin pre partum and 6 week post partum. Intermediate risk patients should receive LMW heparin post partum for 7 days.

\section{Pulmonary hypertension}

Severe pulmonary vascular disease with (in the Eisenmenger syndrome) or without septal defects has long been known to carry the highest risk (maternal mortality $30-50 \%$ ). This is mainly because of a life-threatening further rise in pulmonary vascular resistance due to pulmonary thrombosis or fibrinoid necrosis which develops particularly fast in the peripartum and postpartum periods. In the Eisenmenger syndrome right to left shunting increases during pregnancy because of systemic vasodilatation and right ventricular overload with increased cyanosis and decrease in pulmonary blood flow. Patient with severe pulmonary hypertension should be advised against pregnancy and termination of pregnancy during early pregnancy could be considered. If the pregnancy is to be continued strict monitoring should be done in hospital from second trimester. Foetus should be delivered when viable. If there is intra cardiac shunt such as VSD maintain blood pressure at elevated level to prevent increase in right to left shunt. Mother should be monitored in ICU following delivery to manage impending severe right heart failure.

\section{Complications encountered in} aorta

\section{Coarctation of the aorta}

It is an uncommon association of pregnancy. Coarctation segment commonly sites after the origin of left subclavion artery (Figure 3). There is risk of rupture of the aorta following increased blood pressure secondary to fluid retention and lax connective tissue in aorta. Aortic rupture is commonest reported cause of death in pregnancy following coarctation of the aorta.

\section{Figure 3. CT with 3D reconstruction of coarctation of aorta.}

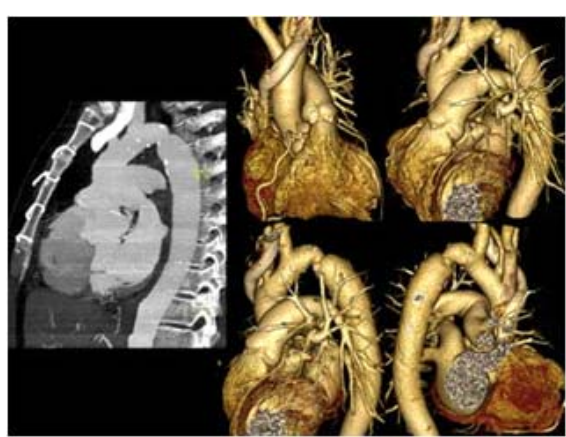

Fetal growth is usually not affected despite of low blood pressure in distal aorta. Preconception correction is the ideal way to prevent complication. During pregnancy restriction of physical activity is the only way of minimizing potentially dangerous surges in blood pressure. A betablocker should be prescribed prevent aortic dilatation and dissection. Surgical correction is indicated in case of uncontrollable systolic hypertension or if heart failure is present. Balloon angioplasty for coarctation is contra-indicated because of the risk of dissection or rupture.

\section{Aortic dissection and Marfans syndrome}

Incidence of Marfans syndrome in general population is 1 in 5000 . It is the most serious dominantly inherited fibrillin-1 deficiency disorder, affecting all systems. Eighty percent of Marfans patients have some cardiac involvement. The majority have mitral valve prolapse with mitral regurgitation and possibly associated arrhythmia. Aortic aneurysm, rupture and dissection are still the commonest causes of death in Marfans syndrome. Pregnancy is a high-risk period and dissection occurring most often in the last trimester or the early postpartum period. Preconception period risk should be assessed in the affected females. Marfans patient with an aortic root diameter of more than 4 $\mathrm{cm}$ should be told that the risk of dissection during pregnancy is $10 \%$. The pros and cons of pregnancy should be fully discussed as well as the alternatives. The risk is lower for pregnancy following elective aortic root replacement for aortic root diameters of $4.7 \mathrm{~cm}$ and over. During pregnancy these patients need to be monitored by echocardiography of the remaining aorta at 6-8 week intervals throughout the pregnancy and for 6 months postpartum. Betablocker therapy should be continued throughout the pregnancy.

\section{Figure 4. CT scan of ascending aorta dissection. Tennis ball sign in ascending.}

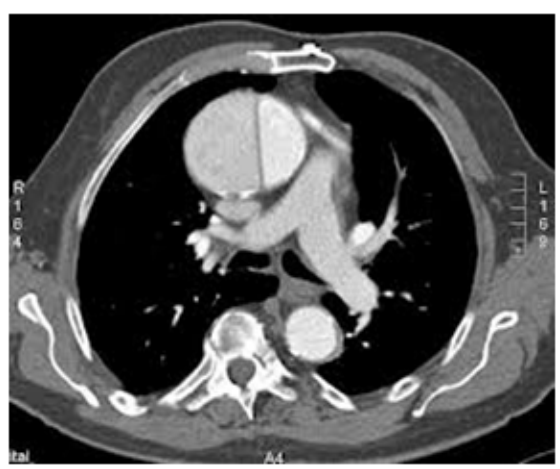

Acute dissection of the ascending aorta during pregnancy is a surgical emergency (Figure 4). Repair with a composite graft is the procedure of choice. Acute dissection with origin beyond the left subclavian artery without involvement of the proximal aorta should be managed medically 
and it can be followed by serial MRI. Progressive dilatation to $5 \mathrm{~cm}$ or more, recurrent pain, or signs consistent with fresh dissection such as the development of organ or limb ischaemia, are all indications for repair. The baby, if viable, should be delivered by caesarean section before going on to bypass. During delivery the second stage should be expedited. The woman may be allowed to labour on her left side or in a semi-erect position to minimize stress on the aorta. If the aortic root diameter is 4.5 $\mathrm{cm}$ or greater caesarean delivery is advised.

\section{Stenotic heart valve disease}

Rheumatic heart valve disease remains a major problem for public health in developing countries. Obstructive and regurgitant valve diseases are common in young pregnant women.

In pregnancy a fixed obstruction, like severe mitral or aortic valve stenosis, may not be able to accommodate the increased cardiac output caused by increased plasma volume. Combined with the drop in peripheral vascular resistance there is a sharp increase in the transvalvular gradient which is poorly tolerated by pregnant mothers. The onset of functional worsening occurs most frequently during the second trimester when the volume reached the peak. For mitral valve it is further worsen by shortened diastolic filling time by tachycardia contributing to further congestion in left atrium.

\section{Mitral stenosis}

In patients with a mitral valve area $<1.5 \mathrm{~cm}^{2}$ (or $1 \mathrm{~cm}^{2} / \mathrm{m}^{2}$ of body surface area) pregnancy carries a risk of pulmonary oedema, congestive heart failure (CHF), arrhythmia and intrauterine growth retardation. In severe mitral stenosis, even if the patient was totally asymptomatic before pregnancy or during the first trimester the mean transmitral gradient and pulmonary artery pressure should be measured by Doppler echocardiography at three and five months and monthly thereafter.

In mothers who have symptoms or estimated systolic pulmonary artery pressure $>50 \mathrm{mmHg}$ medical treatment with beta blockers should be started to prolong the diastolic filling time. Choice of selective agents such as atenolol or metoprolol limits the risk of interaction with uterine contractions. Digoxin also helps to control the heart rate. Diuretics need to be added if signs of pulmonary congestion persist. If the patient still has symptoms and/or pulmonary hypertension percutaneous balloon mitral valvotomy (PMV) has now replaced surgery (Figure 5). PMV is not recommended prophylactically or in patients who have severe mitral stenosis but no pulmonary hypertension and good

\section{Figure 5. Rheumatic mitral valve and balloon valvuloplasty.}
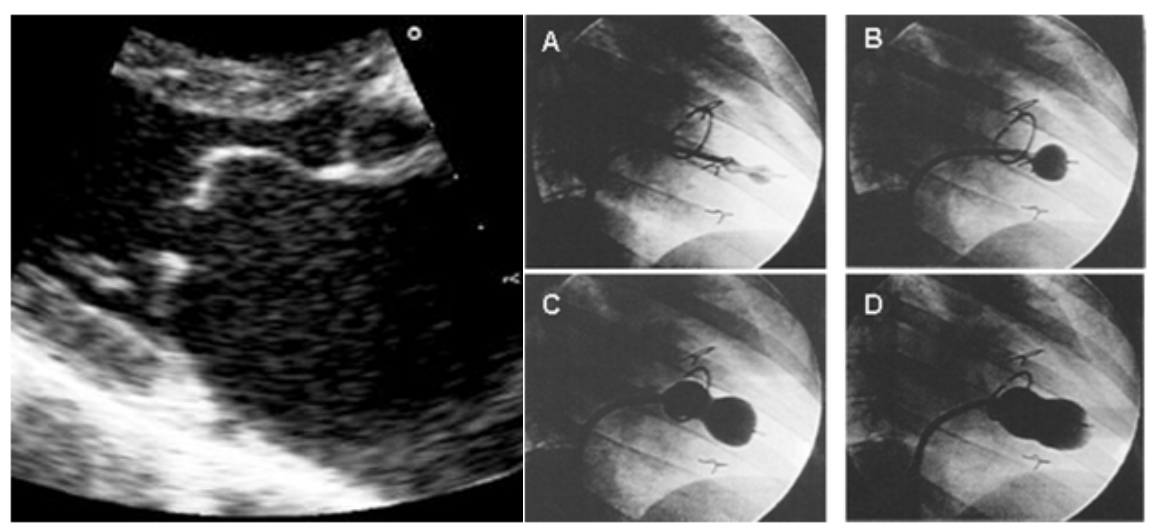

functional tolerance. If valve is not suitable for PMV closed mitral valvotomy has been considered the procedure of choice during pregnancy.

Aorticvalve stenosis / left ventricular outflow tract obstruction (LVOT obstruction)

A fixed outflow tract resistance may not be able to accommodate the increased cardiac output caused by increased plasma volume. This can lead to heart failure with a detrimental rise in left ventricular pressure and pulmonary capillary pressures leading to pulmonary congestion and low output. Mild or moderate aortic stenosis tolerates well during pregnancy. In severe aortic stenosis termination of the pregnancy is recommended. If pregnancy continues monitor systemic pressure and the ECG, as changes can indicate the appearance or worsening of left ventricular overload. Delivery is safe in patients whose functional tolerance is good. During pregnancy if patients remain severely symptomatic, in particular if they have signs of heart failure, aortic stenosis should be relieved before delivery. Aortic balloon valvuloplasty is beneficial to avoid valve replacement surgery. This procedure is best performed in the second trimester when embryogenesis is complete and to avoid any negative effect of ionic contrast agents on the foetal thyroid late in gestation. If calcified aortic valve or there is significant aortic regurgitation surgery is the alternative. Cardiopulmonary by-pass has a foetal mortality of $20 \%$. When fetus is viable deliver the baby by caesarean section before the cardiac surgery.

\section{Pulmonary valve stenosis / RVOT obstruction}

Right ventricular outflow tract (RVOT) obstruction tends to be well tolerated during pregnancy despite the gestational volume overload imposed on an already pressure-loaded right ventricle. However, rarely when the stenosis is severe, pregnancy may 
precipitate right heart failure. Mothers with pulmonary stenosis tolerate pregnancy without events. In severe pulmonary valvular stenosis if mother is symptomatic with right ventricular failure, balloon valvulotomy is the option of choice.

\section{Regurgitant valve lesions}

Unlike obstructive lesions regurgitate lesions are well tolerated during pregnancy. The increase in blood volume and cardiac output will increase the volume overload and worsen the valvular regurgitation but the decrease in systemic vascular resistance promote forward flow and reduces the regurgitant fraction which compensates in part for this. In aortic regurgitation the shortening of diastole due to increase pulse rate in pregnancy also contributes to a reduction in the regurgitant volume. In mitral and aortic regurgitation patients may develop progressive $\mathrm{CHF}$, particularly during the third trimester when volume overload override the compensation by decrease vascular resistance. They need diuretics and vasodilators to reduce afterload even further unless blood pressure is low. Angiotensin receptor antagonists and ACE inhibitors are contraindicated and the only available vasodilators are nitrates and the dihydropyridine calcium-channel blockers like nifidipine. Vaginal delivery may be carried out safely in most patients, even those who have experienced transient heart failure.

\section{Heart valve prostheses}

Prosthetic heart valves pose two main concerns during pregnancy. Firstly, increase risk of valve thrombosis due to hypercoagulable state exists throughout pregnancy. Secondly, treatment with vitamin $\mathrm{K}$ antagonists wafarin cross the placenta and increase the risk of early abortion, embryopathy and prematurity. The overall risk of valve thrombosis seems to be around $5 \%$ in patients who receive vitamin $\mathrm{K}$ antagonists between the sixth and the twelfth weeks, the risk is dose related. During pregnancy vitamin $\mathrm{K}$ antagonists can be used during the second and third trimester. They should be replaced by percutaneous or intravenous heparin at the 36th week to avoid the risk of neonatal intracranial haemorrhage during delivery. The alternative is elective caesarean section at 36 weeks. It is sensible because it minimizes the period on heparin.

There is no consensus regarding treatment during the first trimester. The continuation of a Vitamin K antagonist allows safe and stable anticoagulation for the mother. Recent data suggest that the risk of abortion or embryopathy is very low in patients who take $\leq 5 \mathrm{mg}$ of wafarin per day. The alternative is to use subcutaneous unfractionated heparin during the first trimester, particularly between the sixth and twelfth week particularly in patient who takes $>5 \mathrm{mg}$ of wafarin. This regimen decreases the risk of embryopathy to zero only if heparin is started before the sixth week. Low-molecular weight heparin has advantages over unfractionated heparin as it provides a more stable anticoagulation level. Despite of anticoagulation regimen, patients with a mechanical prosthesis in pregnancy is associated with a maternal mortality between 1 and $4 \%$, mainly due to valve thrombosis while on heparin therapy (Figure 6). In young females with severe valvular heart disease valve repair is beneficial than valve replacement before conception and biological valves should be considered for replacement. Not with standing greater haemodynamic stress vaginal delivery under epidural analgesia is safe in patients with heart valve disease provided they are in stable condition. To shorten the total duration of labour particularly assisting the second stage may be helpful. Caesarean section has the advantage of avoiding the physical stress of labour, but it is not free from haemodynamic consequences related to anesthesia and assisted ventilation. In patients on anticoagulant therapy, heparin should be withdrawn $4 \mathrm{~h}$ before caesarean section or at the onset of labour and resumed 6-12 $\mathrm{h}$ after either surgical or vaginal delivery. Breast-feeding can be encouraged in women taking anticoagulants as heparin is not secreted in breast milk and the amount of warfarin is low. In high-risk patients with previous endo-carditis or heart valve prostheses, prophylactic antibiotic-treatment should be given at the beginning of labour and during delivery.

\section{Figure 6. Prosthetic valve (A) and prosthetic valve thrombosis (B).}

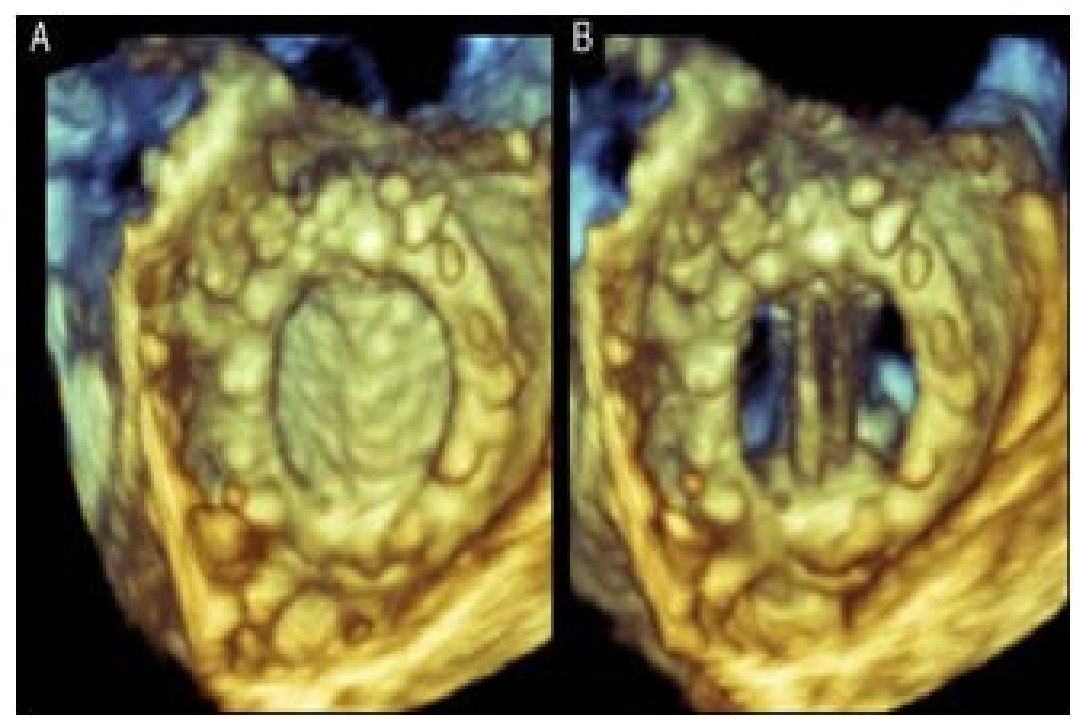




\section{Cardiomyopathy}

\section{Peripartum cardiomyopathy}

Peripartum cardiomyopathy could be suspected in case of unexplained left ventricular systolic dysfunction which is confirmed by echocardiography and develops in the last month prenatally or within five months of delivery. Cardiac biopsy usually shows acute myocarditis if it is performed early after onset. The cause is unknown but possibly an immune reaction to the 'foreign' fetus. The most frequent time of presentation is during the first few days postpartum. Less severe cases require standard therapy for heart failure and their left ventricular function should be followed-up carefully. During pregnancy, ACE inhibitors, ARBs and renin inhibitors are contraindicated because of fetotoxicity. When ACE inhibitors are needed during breastfeeding, benazepril, captopril, or enalapril should be preferred. Hydralazine and nitrates can be used instead of ACE inhibitors/ ARBs for afterload reduction. BBlocker treatment is indicated for all patients with heart failure, if tolerated. Selective drugs (i.e. metoprolol) should be preferred. Anticoagulants are important as the risk of systemic embolism is high. The addition of bromocriptine to standard heart failure therapy has beneficial effects on ventricular $\mathrm{EF}$ and clinical outcome in women with acute severe PPCM. Failure may be fulminating and require inotropes, dopamine and levosimendan can be used if inotropic drugs are needed. Ventricular assist device or even transplantation may be necessary in some patients.

\section{Dilated cardiomyopathy (DCM)}

Patients with DCM should be advised against pregnancy because of the high chance of deterioration both during gestation and peripartum. If becomes pregnant, termination should be advised if the ejection fraction is $<50 \%$ and/or the LV dimensions are definitely above normal. If termination is refused the patient must be seen frequently and LV function checked by echo. For heart failure standard medical therapy should be continued without both ACE inhibitors and angiotensin II antagonists as they are contra-indicated.

\section{Hypertrophic cardiomyopathy}

Hypertrophic cardiomyopathy is an autosomal dominant disorder affecting cardiac myocytes-sarcomere protein. This will cause disorganized myofibriler arrangement. It starts to manifest as a focal involvement in $\mathrm{LV}$ muscle mass in hypertrophic pattern. It commonly affects the septum and sometimes causing obstruction to the left ventricular outflow tract. During the pre conception period in the absence of a family history of sudden death, asymptomatic patients can be told that their risks are low and that pregnancy is usually completed successfully. After the diagnostic echo and an ECG, exercise testing and ambulatory ECG monitoring and genetic counseling are carried out. The genetic risk needs to be discussed including the phenomenon of anticipation, which determines an earlier onset and more severe form in succeeding generations in some families. During pregnancy betablockers should be continued and a small dose of diuretic may help but rest in conjunction with the betablocker to prevent tachycardia is essential in these rare high-risk patients. If atrial fibrillation (AF) develops anticoagulation is imperative preferable with low molecular weight heparin. If new onset $\mathrm{AF}$ fails to revert to sinus rhythm, DC reversion may be necessary after excluding left atrial thrombus. A beta-blocker will help control the ventricular rate in $\mathrm{AF}$ and to prevent recurrence. Patients with persistent arrhythmias, particularly symptomatic ventricular arrhythmias, which have developed during pregnancy, may need amiodarone despite the risk of inducing fetal hypothyroidism. The patient can go through normal delivery with continuation of a beta-blocker and avoidance of systemic vasodilatation as it may cause under filling of $\mathrm{LV}$ cavity and increase LVOT gradient. Any blood loss should be replaced but care should be taken not to cause fluid overload in high-risk patients with very labile left atrial pressures.

\section{Coronary artery disease}

Sudden severe chest pain in a previously fit pregnant woman needs exclusion of dissection of the aorta. If the pain is caused by myocardial infarction it is most likely that spontaneous dissection of a coronary artery has occurred. Coronary artery disease due to atheromatus disease is not very common in pregnancy like in general population. However, the increasing number of older age conception, diabetes, obesity, smoking (active or passive) and hypercholesterolemia makes pregnant women prone to this condition. Some uncommon causes such as Kawasaki disease with coronary aneurysm, coronary arteritis due to autoimmune vascular disease and congenital coronary anomalies complicate coronary artery disease and lead to myocardial infarction during pregnancy. During pregnancy treatment is to provide them with sufficient coronary flow reserve to carry them safely through the pregnancy. Aspirin, beta-blockers and calcium antagonists are sufficient. ACE inhibitors and statins are contraindicated during pregnancy. Exercise testing is important in assessing effectiveness of the therapy. If treatment is suboptimal, percutaneous intervention (PCI) can be performed with care to minimize the radiation dose to the fetus during second trimester. In an acute myocardial infarction, thrombolytics are not to be given although they are only relatively contraindicated in pregnancy. As there is a suspicion of dissection of aorta immediate coronary angiography is performed with a view to PCI and stenting. 


\section{Congenital heart disease}

\section{Shunts}

Patients with small or moderate shunts without pulmonary hypertension such as ASD, VSD and PDA or mild or moderate valve regurgitation benefit from the decrease of systemic vascular resistance that occurs during pregnancy. These patients do not need any intervention.

\section{Cyanotic heart disease}

The commonest cyanotic heart disease is Eisenmenger syndrome due to shunt reversal and tetrology of fallot, as they make it to adult life. The overall maternal mortality is around $2 \%$ with high risk of complications (30\%) such as infective endocarditis, arrhythmias and CHF. The fetal prognosis is also very poor with a high risk of spontaneous abortion (50\%), premature delivery $(30-50 \%)$ and low-for-dates birth weight because maternal hypoxaemia impairs foetal growth. Thromboembo-lism is one of the risks in high-risk pregnancies and the use of pro-phylactic heparin should be consi-dered especially after surgical delivery and in the puerperium. Corrected congenital heart disease patients have good prognosis according to the degree of correction.

\section{Treatment of high-risk patients}

Pregnancy is not recommended. If pregnancy occurs, termination should be advised as the risks to the mother are high (mortality 8-35\%, morbidity $50 \%)$. Physical activity should be restricted and bed rest is recommended if symptoms occur. Oxygen should be given if hypoxaemia is evident. The patient should be hospitalized by the end of the second trimester and low molecular weight heparin administered subcutaneously, as prophylaxis against thromboembolism particularly in cyanotic patients. In severe cyanotic heart disease, monitoring of oxygen saturation is very important. Haematocrit and haemoglobin levels are not reliable indicators of hypoxaemia due to the haemodilution that occurs in pregnancy. If severe hypoxaemia is present and termination of pregnancy is refused some kind of shunt should be implanted if feasible to improve oxygenation.

\section{Tetralogy of fallot}

The risk is high when oxygen saturation is $<85 \%$. The rise in blood volume and venous return to the right atrium with a fall in systemic vascular resistance increases the right to left shunt and cyanosis. Close monitoring of systemic blood pressure and blood gases during labour is needed and any further systemic vasodilatation (drug induced) should be avoided. In patients with significant residual RVOT obstruction, severe pulmonary regurgitation with or without tricuspid regurgitation and/or RV dysfunction, the increased volume load of pregnancy may lead to right heart failure and arrhythmias. This has to be anticipated and treated. All patients with tetralogy should have genetic counseling pre-conception with assessment in case of 22q11 deletion syndrome using fluorescent in situ hybridisation (FISH). In its absence the risk of defects in the fetus is low (about 4\%).

Figure 7. Tetrology-pulmonary stenosis, right ventricular hypertrophy, overriding aorta, ventricular septal defect.

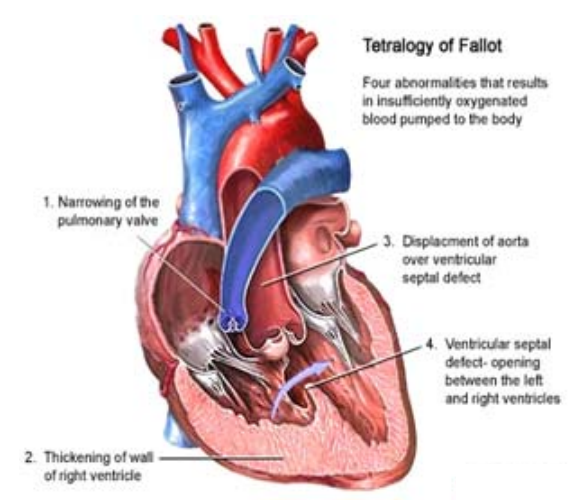

Transposition of great arteries (TGA) - intra-atrial repair

In women in functional class I-II pregnancy is usually well tolerated. Worsening of systemic ventricular function during or shortly after pregnancy occurred in $10 \%$ of the reported cases. They need heart failure therapy.

Transposition of the great arteries congenitally corrected

Women without significant other cardiac defects usually do well but problems can develop through failure of the systemic right ventricle with increasing regurgitation through its tricuspid atrioventricular valve.

\section{Arrhythmias}

Both ectopic beats and sustained arrhythmias become more frequent during pregnancy. They are treated in the same way as outside pregnancy but as conservatively as possible reserving definitive treatment for later if it is safe to do so. All commonly used antiarrhythmic drugs cross the placenta. The pharmacokinetics of drugs is altered in pregnancy and blood levels need to be checked to ensure maximum efficacy and avoid toxicity.

\section{Supra ventricular tachycardia}

Supraventricular tachycardias (SVT) are corrected by vagal stimulation or, failing that, intravenous adenosine rapid bolus. Electrical cardioversion is not contra-indicated and should be used for any sustained tachycardia causing haemodynamic instability and therefore threatening foetal security. Beta blocking drugs with beta-1 selectivity such as metaprolol, bisaprolol are the first choice for prophylaxis. If a class III agent is needed amiodarone is preferable to sotolol. Lesser amounts of amiodarone cross the placenta but long term use can cause neonatal hypothyroidism (9\% of newborns), hyperthyroidism and goiter, so it should only be used when other therapy has failed ${ }^{1}$. Radio 
frequency ablation for AV nodal re-entry or certain AV re-entry tachycardias can, if necessary, be performed during pregnancy with suitable lead shielding and maximal use of echo rather than X-ray fluoroscopy.

\section{Ventricular tachycardia}

Potentially life threatening ventricular tachyarrhythmia (VT) is much less common and should be treated by electrical cardioversion. Beta-1 selective beta-blockers alone, amiodarone alone or the combination may be effective in prevention but if ineffective an ICD will be needed.

\section{Bradycardia}

Symptomatic bradycardia due to sick sinuse syndrome or heart blocks can be treated with pacemaker at any stage of pregnancy. Using echo rather than fluoroscopy as the guide for this procedure is ideal.

\section{Summary}

Managing heart disease in pregnancy needs sufficient knowledge in changing haemodynamics and the application of this knowledge to manage the mother and the fetus safely with limited treatment options available. Multi disciplinary approach involving the obstetrician, cardiologist and anesthetist is vital for a successful outcome.

\section{FURTHER READING}

1. Vera Regitz-Zagrosek, Carina Blomstrom Lundqvist, Claudio Borghi et al. ESC Guidelines on the management of cardio- vascular diseases during pregnancy. European Heart Journal 2011; 32: 3147-97.

2. Pregnancy associated cardiomyopathy : Clinical characteristics and comparison between early and late presentation. Circulation 2005; 111: 2050-5.

3. Warner CA, Williams RG, Bashore TM et al. ACC/AHA 2008 guideline for the management of adult with congenital heart disease. Circulation 2008; 118: e 714-833.

4. De Wilde JP, Rivers AW, Price DL. A review of the current use of magnetic resonance imaging in pregnancy and safety implications for the fetus. Prog Biophys Mol Biol 2005; 87: 335-53.

5. Baumgartner $\mathrm{H}$, Bonhoeffer $\mathrm{P}$, De Groot NM et al. ESC Guidelines for the management of grown-up congenital heart disease (new version 2010). Eur Heart J 2010; 31: 2915-57.

6. Use of antithrombitic agents during pregnancy. Chest 16: $2004 ; 6275-445$. 